\title{
Téoros
}

Revue de recherche en tourisme

\section{Quand les Québécois font du tourisme, chez eux ou ailleurs...}

\section{Marc Laplante}

Volume 4, numéro 2, juillet 1985

Les clientèles touristiques : un tour d'horizon

URI : https://id.erudit.org/iderudit/1080626ar

DOI : https://doi.org/10.7202/1080626ar

Aller au sommaire du numéro

Éditeur(s)

Université du Québec à Montréal

ISSN

0712-8657 (imprimé)

1923-2705 (numérique)

Découvrir la revue

Citer cet article

Laplante, M. (1985). Quand les Québécois font du tourisme, chez eux ou ailleurs... Téoros, 4(2), 12-22. https://doi.org/10.7202/1080626ar d'utilisation que vous pouvez consulter en ligne.

https://apropos.erudit.org/fr/usagers/politique-dutilisation/ 


\title{
Quand les Québécois font du tourisme, chez eux ou ailleurs...
}

\author{
par Marc Laplante, *
}

Partir de chez soi à I'occasion des vacances est la condition première du statut de touriste. Et, généralement, un départ sous-entend une destination. Ce concept, éminamment touristique, sert fréquemment à fabriquer des typologies de clientèles : destination-soleil, départ vers les vieux pays, voyages aux Indes, découvertes de l'Afrique, etc. Pratiquement aussi, pour qui doit vendre des voyages de vacances, le choix des destinations à offrir peut difficilement se faire efficacement sans une bonne connaissance des préférences des clients en cette matière.

Les Québécois, en tant que touristes, peuvent donc être étudiés à partir des destinations de vacances qu'ils choisissent. C'est ainsi que nous les aborderons dans cet article, en distinguant les gens qui voyagent au Québec de ceux et celles qui sortent du territoire national.

Les données sur lesquelles nous nous fonderons pour faire cette étude viennent d'un sondage téléphonique réalisé auprès de 1907 personnes de 18 ans et plus résidant au Québec à I'automne 1982. Les questions analysées ici portaient sur le voyage de vacances réalisé au cours de I'année 1982 Idurant les 12 mois précédant le sondage). Si la personne interrogée avait fait plus qu'un voyage de vacances au cours de cette année lä, elle choisissait de nous décrire le voyage qui représentait le plus ses habitudes touristiques. Dans le cadre de la recherche dont il sera question ici, un départ de vacances signifie: avoir quitté son domicile pour une période de 4 jours de suite ou plus, uniquement pour des vacances. Ainsi défini, le voyage de vacances ne peut inclure les sorties de week-end.

Le sondage, fait avec M. Roger Nadeau de I'Université de Sherbrooke, a donné lieu à plusieurs publications jusqu'à ce jour ${ }^{\text {(1) }}$. Toutefois, certaines analyses ont *Marc Laplante est sociologue es professaur au
département d'études urbaines de ruôM. II remer-
cie Louise Trottier, documentaliste, qui s'est chargée
du traitement statistique des donneves présentées dans cen texte. été approfondies par la suite et quelques données n'ont pas encore été diffusées. Parmi celles-ci, mentionnons ce que nous pourrions appeler les univers socioculturels des diverses clientèles, c'est-àdire, leurs goûts, leurs préférences, leurs attentes et desirs, leurs satisfactions, etc... Nous retiendrons donc ces aspects. rarement étudiés, pour présenter les clientèles de Québécois; mais pour faire des liens avec d'autres recherches, nous tracerons également - mais sommairement - les profils démographiques et socio-économiques de ces mêmes clientèles ${ }^{|2|}$.

\section{Les partants et les autres}

1982 ne fut certes pas une année faste pour les touristes, au Québec comme ailleurs. Chez nous, $34 \%$ des gens (adultes) n'ont pas pris de vacances cette année-lä et $15,4 \%$ n'ont pas quitté leur domicile durant leur période de vacances. Les partants ont constitué alors $50,6 \%$ de la population. Mais, rétrospectivement, ce taux de départ de 1982 est compatible avec celui des années antérieures ${ }^{|3|}$. La situation économique particulière de 1982 s'est probablement exprimée le mieux dans le fait que $61 \%$ des partants sont restés au Québec.
On trouvera ci-dessous un tableau descriptif, très éloquent à propos de la société québécoise et des pratiques touristiques de sa population. Les partants, selon diverses destinations, sont présentés sur l'ensemble de la population.

On constate que sur 100 Québécois adultes, 20 sont sortis du Québec pour des fins de vacances en 1982. On pourrait relever, de plus, que $5 \%$ à peine des Québécois, cette année-là, ont peut-être connu une expérience touristique assez marquée en allant soit en Europe, au Mexique, dans les Caraibes, soit dans des régions étasuniennes moins fréquentées que la Floride ou la côte est.

La réalité des pratiques touristiques globales des Québécois en une année n'est donc pas des plus exotiques à première vue. La destination Québec, visite d'une autre région que la sienne, est nettement la pratique la plus répandue dès qu'on prend des vacances.

Tableau 1

Répartition de la population adulte du Québec selon les utilisations du temps de vacances, 1982. (Total : 1907 personnes interrogées)

a) N'ont pas pris de vacances en 1982

b) Ont pris des vacances, sans partir

c) Sont partis :

- au Québec dans leur région

- au Québec, dans une autre région

- en Ontario

- dans les Maritimes

- ailleurs au Canada

- aux Etats-Unis: en Floride

$23,8 \%$

$3,2 \%$

$1,7 \%$

$0,4 \%$

$5,1 \%$

sur la côte est

$4,3 \%$ ailleurs

- en Europe

$2,1 \%$

- au Mexique et Caraibes

- ailleurs dans le monde 
II ne faudra pas perdre de vue également le groupe des partants qui restent dans leur région. Des catégories du tableau 1 . nous pouvons retenir 5 grandes destinations touristiques qui expriment autant de types d'expériences différentes de voyage de vacances. Le tableau 2, ci-après, énumère ces divers groupes de partants et donne les fréquences relatives pour chacun.

Voilà donc, grossièrement définies, cinq clientèles de touristes québécois; nous verrons d'abord les profils de base de chacune dessines avec des donnes géographiques, démographiques et socioéconomiques: par la suite, la description des univers socio-culturels propres à chacune de ces grandes clientéles nous introduira aux expériences de vacances proprement dites.

\section{Les traits marquants des clientèles québécoises selon les grandes destinations}

Le choix des destinations de vacances est lié principalement aux conditions socioéconomiques des ménages et des individus qui font du tourisme: plus les revenus bruts annuels des ménages sont élevés, plus il y a éloignement de la région de résidence habituelle. Les moins fortunés voyagent plus dans leur région; s les revenus augmentent un peu, on va dans d'autres régions du Québec: avec des ressources financières se situant aux alentours des movennes nationales (plus ou moins 25,000\$), le touriste québécois passe davantage les frontières provinciales, vers les Maritimes, l'Ontario ou I'Ouest canadien; il faut atteindre au moins $30,000 \$$ par annèe pour se diriger vers les USA et $40,000 \$$ pour visiter d'autres pays. Les corrélations entre ces choix de destinations touristiques et les revenus sont nettement plus fortes que toutes les autres relations statistiques; on observe évidemment que les gens les plus scolarisés s'éloignent davantage de leur lieu de résidence; de même, les travailleurs des professions liberrales, des secteurs de la santé et surtout de l'enseignement, les cadres, les administrateurs, etc., partent également davantage vers de lointaines destinations mais nous avons observé, en un premier temps, de fortes liaisons statistiques entre les catégories de scolarité, de profession et de revenus.

Nous avons noté également que les ménages composés d'une seule personne partent beaucoup plus que les autres hors du continent nord-américain, que les ménages de 2 personnes vont davantage vers les USA et les provinces canadiennes, que ceux de 3 ou 4 personnes visitent plus les régions québécoises et finalement, que les menages nombreux restent davantage dans leur région de résidence.
Ces données sont recoupées par les corrélations avec le fait d'avoir - ou non des enfants, avec le fait d'avoir son emploi depuis peu de temps, etc...

Toutefois, si on considère le choix des destinations selon l'äge des répondants, on constate, avec les données du tableau 3. ci-dessous, que ce facteur est aussi étroitement lié aux préférences des partants. Les très jeunes touristes (18-19 ans) restent nettement plus dans leur propre région que les autres groupes d'äge; les 20-39 ans voyagent plus au Québec; les 40-54 ans vont vers les U.S.A., avec les 65 ans et plus; enfin, les 55-64 ans partent davantage vers le Canada et surtout vers les autres pays. La destination de vacances, pour ceux et celles qui partent, semble alors principalement influencée par le cycle de vie: âge, situation matrimoniale, présence ou non d'enfants, nombre et age de ces derniers, veuvage, etc...

Ces deux catégories de facteurs, le cycle de vie et les conditions socio-économiques, sont par ailleurs fort reliées dans les situations réelles: à revenus annuels égaux un ménage de 2 ou 3 enfants à charge est moins capable de partir loin de chez lui pour un voyage de vacances qu'un couple sans enfant. L'elloignement implique évidemment un investissement plus grand pour le transport; mais aussi, plus on s'êloigne de chez soil, plus généralement on va vers des lieux socialement inconnus; ceci implique des investissements plus grands pour l'hêbergement, la restauration et même l'animation de vacances.
Ces résultats ne surprendront pas les intervenants touristiques habitués à tenter de satisfaire les demandes des clientèles québeccoises. Nous nous attarderons surtout à décrire des univers socio-culturels qui sont spécifiques à ces clientèles; ceci nous permettra d'une part, de comprendre que les différences économiques ne rendent compte que d'une partie de la réalité et d'autre part, de réaliser combien, souvent, I'encadrement et l'animation des touristes pourraient s'appuyer sur des attentes connues et assez faciles à satisfaire. Auparavant toutefois, nous voulons souligner une autre corrélation dont le résultat sera apprécié par les acteurs régionaux. Les choix des Québécois en matière de destination touristique varient énormément selon les régions (A.T.R.):

a) voyagent nettement plus dans leur propre région, par ordre, les gens des Laurentides, de l'Outaouais, du Bas StLaurent et de la Gaspésie, du Saguenay Lac St-Jean, de Duplessis et de Charlevoix:

b) voyagent nettement plus dans les autres régions du Québec (que la leur) les gens de Duplessis, Charlevoix, Iles-de-laMadeleine, Saguenay, Lac St-Jean, Bas St-Laurent, Abitibi et Gaspésie;

c) voyagent nettement plus dans les provinces canadiennes: les gens d'AbitibiTémiscamingue, de l'Outaouais, de Laval, des environs de Québec (I'A.T.R. de Québec moins la C.U.Q. ) et de MontréalOuest.

Tableau 2

Les partants, par grande destination. Québec, 1982.

A) au Québec, même région

B) au Québec, autre région

C) au Canada (Québec exclu)

D) aux U.S.A.

E) ailleurs dans le monde

\begin{tabular}{crrrr} 
& \multicolumn{1}{c}{$N$} & & \multicolumn{1}{c}{$\%$} \\
\cline { 2 - 2 } & 158 & & 16,5 \\
& 420 & & 43,9 \\
& 103 & & 10,8 \\
& 204 & & 21,3 \\
& 71 & & 7,4 \\
TOTAL & 956 & & 100,0
\end{tabular}

Tableau 3

Destinations de vacances selon l'åge (en \%)

\begin{tabular}{|l|c|c|c|c|c|}
\hline $\begin{array}{l}\text { Groupes } \\
\mathrm{d}^{\prime} \text { âge }\end{array}$ & $\begin{array}{c}\text { Québec } \\
\text { mềme } \\
\text { région }\end{array}$ & $\begin{array}{c}\text { Québec } \\
\text { autres } \\
\text { régions }\end{array}$ & USA & Canada & $\begin{array}{c}\text { Autres } \\
\text { pays }\end{array}$ \\
\hline $18-19$ ans (43) & 30 & 42 & 9 & 14 & 5 \\
$20-39$ ans (542) & 14 & 50 & 18 & 10 & 8 \\
$40-54$ ans (219) & 20 & 36 & 30 & 8 & 6 \\
$55-64$ ans (93) & 22 & 27 & 22 & 17 & 12 \\
65 ans et plus (60) & 10 & 45 & 25 & 13 & 7 \\
\hline TOTAL (957) & 17 & 44 & 21 & 11 & 7 \\
\hline
\end{tabular}

$100 \%$ 
d) voyagent nettement plus vers les U.S.A. : les gens de Montréal-Ouest. Montréal-Est, Richelieu-Rive-Sud, Pays de l'érable, Laval, Montréal-Ville et Mauricie;

e) voyagent nettement plus dans les autres pays (hors Canada-USA), les gens de Montréal-Ouest, Montréal-Ville, Montréal-Est, Richelieu-Rive-Sud et llesde-la-Madeleine.

Les régions touristiques reflètent d'abord les différences socio-économiques déjà étudiées; elles expriment également l'influence d'un facteur majeur ; I'urbanisation. Au Québec, contrairement à des idées recues, on note un fort mouvement dans les déplacements de vacances, des petites agglomérations vers les plus grandes. Ainsi, beaucoup de gens des lles-de-la-Madeleine et de la Gaspésie choisissent Montréal comme destination; ceci est vrai presque également pour les gens du Saguenay Lac St-Jean et de l'Abitibi-Témiscamingue; on note aussi que les gens de l'Outaouais vont vers la ville de Québec ${ }^{(4)}$.

En arrière-plan de ces déplacements, nous pensons constamment aux relations parentales: les familles des régions éloignées ont dû s'éparpiller sur le sol québécois : les vacances deviennent un temps de retrouvailles; par exemple, un fort pourcentage de gens de Charlevoix se dirigent vers le Saguenay Lac St-Jean et beaucoup de gens de Québec vont vers le Bas St-Laurent. Par ailleurs, les gens des milieux très urbanisés du Québec, et surtout les résidents de Montréal louest, centre, est) partent vers d'autres pays que le Canada ou les USA. La destination étasunienne est préférée par les urbains périphériques IMontréal-Est, Laval, RichelieuRive-Sud, Pays de l'Érable, Lanaudière et Laurentides).

Nous n'oublions pas que les plus hauts revenus se trouvent dans les plus grandes villes (Montréal-Ouest notamment), qu'à revenus égaux, les familles plus nombreuses sont davantage localisées hors des grandes agglomerations, etc... Mais le modèle général du mouvement vers le sud, du déplacement des régions peu densément peuplées vers des régions de plus forte densité de population nous oblige à nous interroger plus rigoureusement sur le poids propre des facteurs géographiques (ce que nous ferons prochainementl.

Poursuivons cette exploration en nous concentrant maintenant sur les facteurs socio-culturels.

\section{Habitudes de vacances, attentes et satisfactions touristiques des diverses clientèles de québécois}

\section{Grandes et petites destinations}

Les Québécois qui font du tourisme dans d'autres régions du Québec que la leur vont, par ordre :

- en Gaspésie, Bas St-Laurent

- dans la région de Montréal

- au Saguenay Lac St-Jean

- dans la région de Québec

- dans les Laurentides

- à la ManicBaie James

- en Estrie

- en AbitibiTemiscamingue

$18,2 \%$

- en Charlevoix

4,9

- en Mauricie

3,9

- en Richelieu-

Rive-Sud

- dans Lanaudière

- dans

r'Outaouais

$\frac{1,8}{100,0 \%} \quad(\mathrm{~N}=384)$

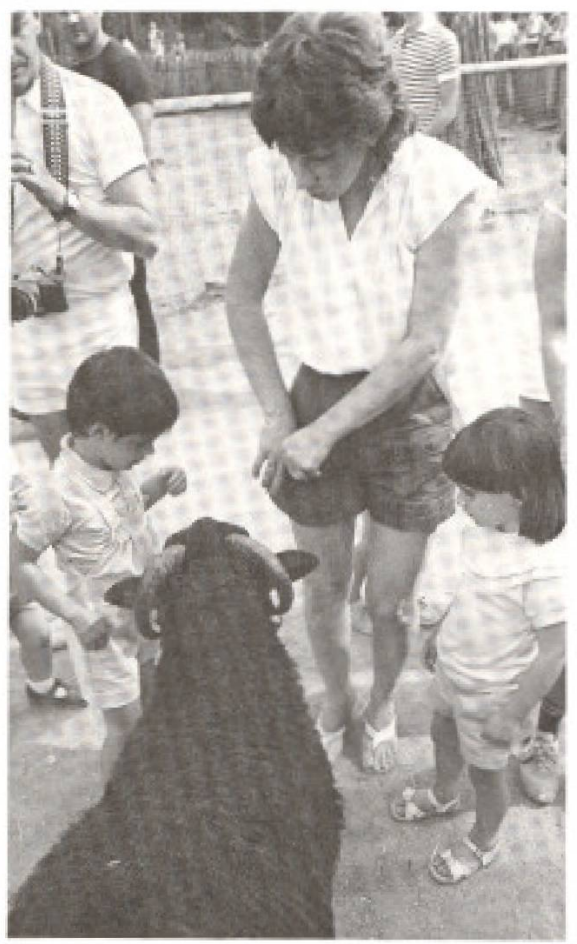

Ả revenus annuels égaux, un ménage de 2 ou 3 enfants à charge est moins capable de partir loin de chez lui pour un voyage de vacances qu'un couple sans enfant.
Les Québécois partant vers les autres provinces canadiennes choisissent, par ordre de préférence :

$\begin{array}{lr}\text { - I'Ontario : } & 54,5 \% \\ \text { - les Maritimes : } & 30,3 \% \\ \text { - I'Ouest } & \\ \text { canadien : } & \frac{15,2 \%}{100,0 \%} \quad(\mathrm{~N}=99)\end{array}$

Les Québéccois en vacances aux U.S.A. wont, par ordre :

\begin{tabular}{lr} 
- en Floride : & $49,5 \%$ \\
- sur la Còte Est : & $40,3 \%$ \\
- ailleurs : & $10,2 \%$ \\
\cline { 2 - 3 } & $100,0 \%$
\end{tabular}$\quad(\mathbb{N}=196)$

Finalement, quand les Québécois partent pour de plus lointaines destinations, ils se dirigent, de préférence :

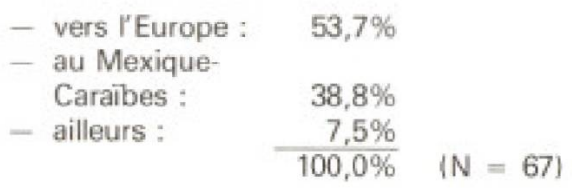

Ces résultats confirment des habitudes assez connues: les provinces limithrophes sont choisies en premier lieu ; ainsi, l'Abitibi-Témiscamingue et I'Outaouais monopolisent les échanges avec l'Ontario car ces régions partagent une longue frontière, ont des rapports de travail et souvent, les réseaux de parents et d'amis s'étendent sur les deux provinces; la Côte-Est des États-Unis, où se trouve la mer la plus proche en été pour les Québécois, est la région étasunienne la plus fréquentée par les gens du Québec, avec la Floride (en hiver): hors de ces territoires voisins, l'Europe reste une destination préférée; la zone Mexique-Caraibes intêresse environ un tiers des touristes au long cours; et le vaste monde reste encore à découvrir par les Québécois.

Ces choix, il faut les voir en relations avec les attentes, les besoins, les valeurs liês au vacances.

\section{Nomades ou sédentaires}

Les styles ou les genres de vacances touristiques s'expriment d'abord dans les façons les plus générales de passer le temps. En tourisme, une dimension s'avère des plus significatives pour caractériser les habitudes: il s'agit du nombre de destinations placées sur l'itinéraire des vacances. Un départ touristique à destination unique devient de la villégiature de vacances /dans une résidence secondaire, un chalet, une station touristique, etc.): un itineraire comptant plusieurs lieux de séjours différents s'apparente davantage au tour original d'où est né le mot tourisme. Nous avons choisi d'appeler ces derniers des nomades alors que les gens

suite a la page 21 


\section{suite de la page 14}

n'utilisant qu'un seul lieu de séjour de vacances furent nommés des sédentaires. Le tableau 4, ci-apres, distingue aussi ces deux grands genres selon que les sédentaires ont fréquenté leur lieu de séjour une ou plusieurs fois et selon le nombre de lieux de séjours différents des nomades.

$66 \%$ des gens qui quittent leur domicile durant leurs vacances mais qui restent dans leur région sont des sédentaires familiers des lieux où ils passent ces vacances. $50 \%$ des gens qui partent vers d'au tres régions du Québec sont aussi des sédentaires familiers ou très familiers avec leur destination. Ce pourcentage est identique pour ceux et celles qui partent vers les U.S.A..

Les voyages au Canada sont routiniers pour $30 \%$ des gens choisissant cette destination; enfin, $17 \%$ seulement des gens qui partent vers de plus lointaines destinations sont des habitués des lieux de vacances choisis.

On notera que l'itinérance - le voyage fait de nombreux déplacements - se pra. tique au Canada et dans les autres pays nettement plus qu'au Québec et aux U.S.A.. La destination étasunienne a beaucoup de traits communs avec la destination québécoise au point de donner raison aux humoristes qui fixent les limites sud du Québec... en Floride!

Vacances nomades ou sédentaires! On admettra aisément que ces distinctions ne sont pas que jeux conceptuels : de vieux du coureur des bois, version québécoise d'Ulysse, le Grec; la terre et les réveries du repos, pour plagier le titre d'une ceuvre du grand philosophe Gaston Bachelard ${ }^{|5|}$; les rapports complexes entre villes et campagnes dans nos sociétés ou l'urbanisation devient galopante, etc... Utilisant nos premières données de recherches, nous avons eu l'occasion d'approfondir un peu archétypes sont ici sollicités: I'aventure

plus les types nomades et sédentaires lors d'une communication en 1984 qui avait reçu une bonne attention d'un public pourtant très proche des touristes: les membres de I'Association technique du tourisme $e^{(6)}$

Le constat initial est le fait que 2 partants sur 3 sont des sédentaires; en second lieu, il s'agit de deux publics distincts, qui s'opposent même sur des faits essentiels :

a) Les sédentaires attendent avant tout de leurs vacances du repos, dans un endroit calme alors que les nomades veulent rencontrer des gens intéressants, dans un milieu excitant, pour expérimenter des choses nouvelles, connaitre l'aventure, voir vivre d'autres gens.

b) Les sédentaires s'adonnent surtout, en vacances, aux sports, au plein air et à la plage, à la lecture; les nomades recherchent davantages les sorties, les spectacles, les visites touristiques, les fêtes, la photographie.

c) Les sédentaires s'entourent des enfants et de la parenté; les nomades sont plutôt avec des amis et des gens rencontrés en voyage.

d) Le voyage inoubliable, pour les sédentaires, tient à la nature observée et visitée; il tient aux rencontres sociales pour les nomades.

e) Les sédentaires sont plus âgés (entre 35 et 54 ans), fort riches financièrement $(40,000$ s et plus), propriétaires de chalet, davantage spécialisés dans le secteur de ladministration, du commerce, de la gérance et des postes de direction: les anglophones y sont sur-représentés. Les nomades se trouvent plutôt parmi les 18-34 ans avec des revenus annuels d'environ 30,000 s, propriétaires de roulotte, plus francophones, membres de professions libérales du secteur de l'enseignement et de la santé.

Tableau 4

Vacances sédentaires ou nomades, selon les destinations (en \%)

\begin{tabular}{|c|c|c|c|c|c|c|}
\hline \multirow[t]{2}{*}{ Destinations } & \multicolumn{2}{|c|}{ Sédentaires } & \multicolumn{4}{|c|}{ Nomades } \\
\hline & $\begin{array}{l}\text { En lieu } \\
\text { très } \\
\text { connu } \\
\text { (a) }\end{array}$ & $\begin{array}{l}\text { En lieu } \\
\text { connu } \\
\text { (b) }\end{array}$ & $\begin{array}{l}\text { En lieu } \\
\text { nou- } \\
\text { veau } \\
\text { (c) }\end{array}$ & $\begin{array}{l}1 \text { ou } 2 \\
\text { desti- } \\
\text { nations }\end{array}$ & $\begin{array}{l}3 \text { ou } 4 \\
\text { desti- } \\
\text { nations }\end{array}$ & $\begin{array}{l}5 \text { ou plus } \\
\text { destina- } \\
\text { tions }\end{array}$ \\
\hline $\begin{array}{l}\text { Québec : Même } \\
\text { région (159) }\end{array}$ & 53 & 13 & 14 & 7 & 6 & 6 \\
\hline $\begin{array}{l}\text { Québec : Autres } \\
\text { régions }(424)\end{array}$ & 43 & 7 & 16 & 9 & 15 & 10 \\
\hline U.S.A. (204) & 31 & 19 & 21 & 8 & 11 & 10 \\
\hline Canada (106) & 21 & 9 & 17 & 7 & 19 & 26 \\
\hline Autres pays $(74)$ & 8 & 9 & 31 & 14 & 11 & 28 \\
\hline TOTAL (967) & 37 & 11 & 18 & 9 & 13 & $12: 100 \%$ \\
\hline
\end{tabular}

a) lieu fréquenté quatre fois (ou plus) avant les vacances de 1982

b) lieu fréquenté de 1 à 3 fois avant les vacances de 1982

c) lieu fréquenté pour la 1ère fois en 1982. f) Finalement, comme nous l'avons vu au tableau 4 , les sédentaires restent davantage dans leur région, au Québec ou aux USA et fréquentent plusieurs fois les mêmes lieux; les nomades vont plus loin et circulent beaucoup.

Compte tenu des différences d'äge entre ces deux publics, nous pouvons nous interroger, comme nous l'avons fait lors de notre conférence, sur l'évolution possible d'un groupe à l'autre avec le temps: les nomades, en vieillissant, deviendront-ils des sédentaires? Ils seront plus riches, auront atteint des postes de direction, auront vu beaucoup de pays, seront plus fatigués, etc. Rêveront-ils de lieux calmes, de gens familiers, de retour dans la nature? II nous faudrait connaitre, pour répondre à ces questions, les habitudes de vacances des sedentaires d'aujourd'hui, il y a 5, 10 ou 20 ans passés. D'autres études devront alors scruter ces sujets.

Le rappel des principaux résultats de la comparaison entre les sédentaires et les nomades simplifie notre présentation des profils socio-culturels de chacune des cinq grandes clientèles de québécois. En effet, les destinations: Québec/même région, Québec/autres régions et U.S.A. étant choisies majoritairement par des sédentaires familiers ou très familiers avec leur lieux de séjour, il faut s'attendre à trouver plusieurs ressemblances entre les clientêles de ces 3 destinations et celle des sédentaires; réciproquement, les touristes vers le reste du Canada ou les autres pays du monde devraient avoir des parentés avec la clientèle des nomades décrite cihaut. Le tableau synoptique 1, Ivoir à la page 22), confirme ces hypothèses :

Ce genre de bilan exige qu'on s'y attarde car il peut être plus éloquent encore par ses sous-entendus que par ses résultats explicites. II nous faut être bref ici et laisser au lecteur le soin de poursuivre l'interrogation.

a) La destination qui est la plus difficile à profiler est justement celle qui occupe le plus de gens : la visite du Québec, hors de sa propre région. Ce tourisme est familial et on le croit capable de favoriser le rapprochement entre les membres de la famille; il est assez satisfaisant; on s'adonne un peu plus au plein air à cette occasion. Mais on y fait moins de plage, de bateau, moins de visites touristiques, on passe moins de temps avec les amis ou les rencontres de vacances. Ceci nous laisse croire que ces voyages au Québec durant les vacances accordent beaucoup de temps à la parenté (c'est la seule destination pour laquelle le temps passé avec les parents differe de l'ensemble) mais que ces rencontres ne sont pas nécessairement très réjouissantes: la vie quotidienne change peu, l'anticipation de ces 
vacances ne provoque aucun sentiment spécial, les attentes sont imprécises lon ne demande pas que le climat soit plus agréable...J, etc... La visite parentale serait-elle un devoir de vacances? Ou alors, peut-être, ces retrouvailles entre parents apportent des satisfactions et favorisent des activités de vacances que notre enquête n'a pas su saisir?

b) Le Canada, à l'exception du Québec. et les autres pays du vaste monde, à l'exception des U.S.A., sont choisis et fréquentés à peu près par le même monde et de la même façon. Mais le Canada n'a pas l'atout climatique et n'est pas choisi avec des attentes bien prononcées. Comme on en revient très satisfait, comme on s'est assez dépaysé, sans faire des folies comme dans les autres pays, cette destination finalement est peut-être le terrain de pratique des Québécois à s'éloigner du village natal, avant de partir vers d'autres cultures, d'autres civilisations. Car le choix des U.S.A. au fond, reste essentiellement une affaire climatique; aux Etats-Unis, c'est comme ici, la mer chaude en plus en été et la chaleur en hiver. L'expérience des Québécois à partir de chez eux commencerait alors avec un voyage dans les Maritimes, en Ontario ou dans les Rocheuses $113 \%$ des $18-29$ ans choisissent le Canada contre $10 \%$ pour l'ensemble des partants).

L'univers socio-culturel des touristes, l'ensemble complexe de leurs désirs et besoins, des factons de les satisfaire, l'expression mème des degrés ou niveaux de satisfaction, toutes ces dimensions qui peuvent éclairer les choix et les comportements observables ne se laissent pas saisir aisément.

Pourtant, il faudra comprendre de mieux en mieux et rapidement ce qui se passe a l'arriere-plan des décisions de vacances et de voyages de vacances. Dire aujourd'hui que les Québécois forment des clientèles touristiques exigeantes serait une erreur; dans ce domaine, encore nouveau pour la grande majorité des gens, les clients prennent, souvent aveuglément, ce qu'on leur propose! L'idée qu'ils pourraient peut-être avoir beaucoup plus pour les mêmes investissements ne les occupe peut-être pas du tout : vacances et tourisme sont des petites folies pour les. quelles on se voit mal négocier le meilleur rapport: coût-bénéficel Cette mentalité vaut encore mais nous voyons aussi que les plus jeunes, dans les milieux urbains, plus instruits ont des attentes plus variees et mieux articulếes et réalisent des projets plus personnels sans attendre d'avoir bien capitalisé leurs revenus.

Voilà des signes de temps nouveaux pour le tourisme au Québec.
Tableau synoptique 1

Besoins, attentes, statisfactions de vacances, selon les clienteles par grandes destinations

\begin{tabular}{|c|c|c|c|c|c|c|c|}
\hline \multicolumn{3}{|c|}{ Elements socio culturels } & \multirow{2}{*}{$\begin{array}{l}\text { QUEBEC } \\
\text { Mämé } \\
\text { région } \\
++ \\
-+\end{array}$} & \multirow{2}{*}{$\begin{array}{l}\text { QUEBEC } \\
\text { Autres } \\
\text { region } \\
-\end{array}$} & \multirow{2}{*}{$\begin{array}{l}\text { U.3.A. A. } \\
+++\end{array}$} & \multirow{2}{*}{$\begin{array}{l}\text { CANADA } \\
--\end{array}$} & \multirow{2}{*}{$\begin{array}{l}\text { AUTAES } \\
\text { PAYS } \\
\begin{array}{l}-+ \\
++ \\
++ \\
++\end{array}\end{array}$} \\
\hline & $\begin{array}{l}\text { Thilfertes envers } \\
\text { la destination }\end{array}$ & 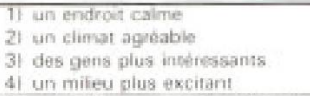 & & & & & \\
\hline & Pantis avec qui? & 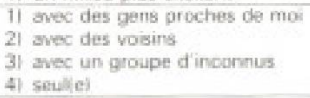 & + & & $\begin{array}{l}+ \\
+ \\
-\end{array}$ & & ++ \\
\hline el & I Satisfaction atuerale & $\begin{array}{l}\text { Besauboub } \\
\text { Ássez } \\
\text { Plus ou maing, gu peu }\end{array}$ & . & - & & + & ++ \\
\hline & $\begin{array}{l}\text { Sentments awant } \\
\text { le depart: }\end{array}$ & 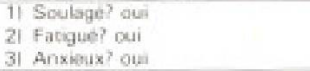 & + & & - & - & $\begin{array}{l}- \\
-- \\
+\end{array}$ \\
\hline & $\begin{array}{l}\text { 1. Habiudes quatidierves: } \\
\text { changees en vacances } \\
\text { |nbponges : BEAUCOUP!) }\end{array}$ & $\begin{array}{l}\text { 11 de mangen } \\
\text { 21 de dormir } \\
\text { 3i de rencontres of aris } \\
\text { 4) de vie artistique } \\
\text { sil de boogson alopoliques }\end{array}$ & + & + & $\begin{array}{l}- \\
- \\
-\end{array}$ & & $\begin{array}{l}++ \\
-+ \\
+ \\
++ \\
++\end{array}$ \\
\hline in & Activitús pratiquetes & 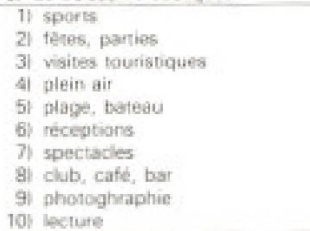 & $\begin{array}{l}++ \\
- \\
-+ \\
+ \\
+- \\
-- \\
--\end{array}$ & -- & $\begin{array}{l}+ \\
+- \\
++ \\
-- \\
+ \\
+ \\
+\end{array}$ & 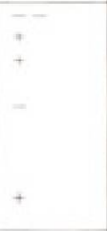 & $\begin{array}{l}-- \\
++ \\
++ \\
-+ \\
++ \\
++ \\
+++ \\
++ \\
+\end{array}$ \\
\hline & $\begin{array}{l}\text { Aver qui on a passed le } \\
\text { plus de temps en vacatnces }\end{array}$ & 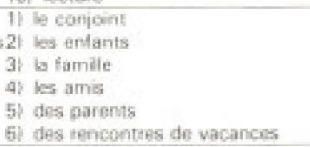 & + & $\begin{array}{l}+ \\
+ \\
+ \\
+ \\
+\end{array}$ & + & + & $\begin{array}{l}-- \\
- \\
++ \\
++\end{array}$ \\
\hline & $\begin{array}{l}\text { Bescoin qu' on lente de } \\
\text { sarisfare par des } \\
\text { wasances }\end{array}$ & 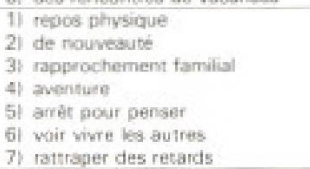 & $\begin{array}{l}+ \\
- \\
- \\
+ \\
+\end{array}$ & + & - & $\begin{array}{l}+ \\
+ \\
+ \\
+\end{array}$ & $\begin{array}{l}- \\
++ \\
-- \\
++ \\
- \\
++ \\
-\end{array}$ \\
\hline & Potterences & $\begin{array}{l}\text { a) Vacances Iangues } \\
\text { b) Aller proche de chez soi } \\
\text { c. Alles dare un leu nouveau }\end{array}$ & + & & $\stackrel{+}{+}$ & $\begin{array}{l}+ \\
+ \\
+\end{array}$ & - \\
\hline & Oue feriez-wous d'un gain & Je le consacnerais di des & $1-=$ & $=$ & + & & + \\
\hline
\end{tabular}

Symboles: +++ ou $-\ldots-$ : différences trẻs significatives, statistiquement entre cette clientèle et

++ ou - - : l'ensemble des partants

+ ou - : différences réelles mais moins marqués entre cette clientèle et

l'ensemble des partants

\section{Rệférences}

(1) Parmi les plus importants tecrits bases sur cette recherche qui a rocu des subventions F.C.A.C. en 1982 et 1983, mentionnons

LAPLANTE, Marc, assiste de Jacques Luneau et Louise Trottier : Gens qui partent et gens qui restent - los grandes vacances au Quebec, Montréal. Uniusrsité du Quebesé is Montréal. Département d'Etudes Urbaines, collection : études urbaines - études touristiques, 1983, 123 pages (épuise).

NADEAU, Roger, Non-vacenciers et vacanciers non-partants au Québec. 1982 : profils comparês, Université de Sherbrooke, Departe ment de Geographie, Bulletin de recherche numeros 71-72, février 1994, 109 pages.

(2) La présentation descriptive de ces profils et de ces univers socio-cultunels exigerait d'ätre accompagnese d'un grand nombre de tableaux statistiques. Nous n'en awons retenu que quelques-uns pour ne pas alourdir indument le présent texte. Les auteurs fourniront, sur demande, les donnees de base non encore publietes et utilisees ici pour liner leurs conclusions. Une publication prochaine de Marc: Laplante: Tourisme et Sociêté, integrara tous bes resultats a des etudes plus recontes faites au Québec sur les pratiques touristiques des diverses clientelves.

(3) Voir : LAPLANTE, Mare : La demande tounistque, in : Foger NADEAU : Le tourisme, aspects théoriques et pratiquess au Québec. Montrétal, Sodilis, 1982, pp. 77 aे 104.

(4) NADEAU, Roger et STE-MARIE, Suzanro: Facteurs infuencant hes destinations do vacances des Quebécois en 1982. ACFAS, communications au 52 e congress, Universite Laval, mai 1984.

(5) 4e edition. Paris, Librairie Jose Corti, 1965.

(6) Conference au Conqres de I.A.T.T. 3 mars 1984, Chateau Fromtenac, Quebec: Profil du touriste des anntes forures. 